\title{
Ueber einige bestimmte Integrale.
}

( Von Herrn H. Weber in Heidelberg.)

Die Integrale, mit welchen sich der vorliegende Aufsatz beschäftigt, enthalten die Besselschen Functionen $\boldsymbol{J}^{(h)}(\boldsymbol{z})$ von beliebiger Ordnung und bilden insofern eine Verallgemeinerung der von Herrn Lipschitz gefundenen Integrale $^{*}$ ) und eine Erweiterung der durch die Resultate von Herrn Lipschitz dargethanen Analogie dieser Functionen mit den trigonometrischen Functionen, wiewohl einige der von mir gefundenen Integrale ihr Analogon unter den trigonometrischen Functionen nicht finden. Die Methode, durch welche man zu diesen Integralen gelangt, beruht auf der Anwendung einer merkwürdigen Formel, welche den Werth eines dreifachen Integrals angiebt, welches eine beliebige nur gewissen Stetigkeitsbedingungen unterworfene Lösung einer partiellen Differentialgleichung enthält. Auf diese Formel wird man naturgemäss geführt durch eine sehr einfache physikalische Betrachtung: Ist nämlich in einem allseitig unbegrenzten Medium eine beliebige anfängliche Temperaturvertheilung $\boldsymbol{\Phi}(\boldsymbol{x} y \boldsymbol{z})$ gegeben, so erhält man mit Hülfe des bekannten Laplaceschen Integrals das Gesetz der Bewegung der Wärme, d. h. die Lösung der Differentialgleichung:

in der Form:

$$
\frac{\partial u}{\partial t}=\frac{\partial^{2} u}{\partial x^{2}}+\frac{\partial^{2} u}{\partial y^{2}}+\frac{\partial^{2} u}{\partial z^{2}}
$$

$$
u=\frac{1}{\pi^{\frac{3}{2}}} \int_{-\infty}^{+\infty} \int_{-\infty}^{+\infty} \int_{-\infty}^{+\infty} d \alpha d \beta d \gamma \Phi(x+2 \alpha \sqrt{ } t, y+2 \beta \gamma / t, z+2 \gamma \sqrt{ } t) e^{-\alpha^{3}-\beta^{2}-\gamma^{2}} .
$$

Nimmt man aber an, die Function $\Phi(x y z)$ sei so beschaffen, dass sie mit ihren ersten Differentialquotienten im ganzen unendlichen Raum stetig ist und gleichzeitig der Differentialgleichung

$$
-\boldsymbol{m}^{2} \boldsymbol{\Phi}=\frac{\partial^{2} \Phi}{\partial x^{2}}+\frac{\partial^{2} \Phi}{\partial y^{2}}+\frac{\partial^{2} \Phi}{\partial z^{2}}
$$

genügt, so erhält man eine Lösung der Wärmegleichung, welche gleichfalls der Bedingung des Anfangszustandes genügt, in der Form:

$$
u=e^{-m^{2} t} \boldsymbol{\Phi}(x y z) \text {. }
$$

*) Bd. 56 dieses Journals. 
Giebt man nun zu, dass die Wärmeprobleme auch für unbegrenzte Medien bei gegebenem Anfangszustand immer nur eine einzige Lösung zulassen, so müssen unter der über $\boldsymbol{\Phi}$ gemachten Voraussetzung die beiden Ausdrücke für $u$ übereinstimmen, woraus die oben erwähnte Formel folgen würde.

Abgesehen davon aber, dass der Beweis der Eindeutigkeit der Wärmeprobleme für unbegrenzte Medien meines Wissens noch nicht hinlänglich geführt ist, wäre ein directer Beweis dieser Formel auch darum wünschenswerth, weil dieselbe offenbar mit dem Wärmeproblem nichts $\mathrm{zu}$ thun hat und die Zeit nur als einen Parameter enthält. Da ausserdem der directe Beweis eine beträchtliche Verallgemeinerung der Formel zulässt, so werde ich zunächst mit einer anderen Ableitung derselben beginnen und dann die oben erwähnte Anwendung auf die Besselschen Functionen machen.

\section{§. 1.}

Wenn man in der Gleichung:

$$
\text { (1.) } \quad-m^{2} \Phi=\frac{\partial^{2} \Phi}{\partial \xi^{2}}+\frac{\partial^{2} \Phi}{\partial \eta^{2}}+\frac{\partial^{2} \Phi}{\partial \zeta^{2}}
$$

Polarcoordinaten einführt, indem man setzt:

$$
\begin{aligned}
& x-\xi=r \sin \vartheta \sin \varphi, \\
& y-\eta=r \sin \vartheta \cos \varphi, \quad \cos \vartheta=\mu, \\
& z-\zeta=r \cos \vartheta, \quad
\end{aligned}
$$

so nimmt dieselbe nach bekannten Regeln die Form an:

$$
-m^{2} \boldsymbol{\Phi}=\frac{1}{r^{2}}\left\{\frac{\partial}{\partial r}\left(r^{2} \frac{\partial \Phi}{\partial r}\right)+\frac{\partial}{\partial \mu}\left(\left(1-\mu^{2}\right) \frac{\partial \Phi}{\partial \mu}\right)+\frac{1}{1-\mu^{2}} \frac{\partial^{2} \Phi}{\partial \boldsymbol{\varphi}^{2}}\right\} .
$$

Legt man nun um den Mittelpunkt $x y z$ mit dem Radius $r$ eine Kugelfläche, in deren Nähe die Function $\Phi$ mit ihren ersten Differentialquotienten als endlich, stetig und eindeutig vorausgesetzt wird, so lässt sich der mittlere Werth dieser Function auf dieser Kugelfläche finden. Setzt man nämlich

$$
\omega=\int_{-1}^{+1} \int_{-\pi}^{+\pi} \Phi d \mu d \varphi
$$

und berücksichtigt, dass:

$$
0=\int_{-1}^{+1} \int_{-\pi}^{+\pi} d \mu d \varphi\left\{\frac{\partial}{\partial \mu}\left(\left(1-\mu^{2}\right) \frac{\partial \Phi}{\partial \mu}\right)+\frac{1}{1-\mu^{2}} \frac{\partial^{2} \Phi}{\partial \varphi^{2}}\right\},
$$

weil nämlich der Voraussetzung der Eindeutigkeit zu Folge $\frac{\partial \Phi}{\partial \varphi}$ für $\varphi=-\pi$ 
und $\varphi=+\pi$ und denselben Werth von $\mu$ denselben Werth annehmen muss, so erhält man durch Integration der Gleichung (2.) über die ganze Kugelfläche:

oder

$$
-m^{2} \omega=\frac{1}{r^{2}} \frac{\partial r^{2} \frac{\partial \omega}{\partial r}}{\partial r}
$$

$$
\frac{\partial^{2} r \omega}{\partial r^{2}}=-m^{2} r \omega
$$

woraus man durch Integration in Bezug auf $r$ erhält:

$$
\text { (3.) } \omega=\frac{1}{r}\{A \sin m r+B \cos m r\} \text {, }
$$

wo die Constanten $A, B$ nur noch von den Coordinaten $x y z$ des Mittelpunkts der Kugel abhängig sein können. Nimmt man nun an, dass die Function $\Phi$ die vorausgesetzten Stetigkeitsbedingungen im ganzen Innern der Kugelfläche erfülle, so muss die Formel (3.) auch noch gültig sein für $r=0$, und da $\omega$ für $r=0$ nicht unendlich werden soll, so muss $B=0$ sein. Setzt man nun $r=0$, so wird

folglich :

$$
\omega_{0}=\Phi(x y z) 4 \pi
$$

$$
A=\frac{4 \pi}{m} \Phi(x y z)
$$

also :

$$
\text { (4.) } \quad \omega=\frac{4 \pi}{m} \Phi(x y z) \frac{\sin m r}{r} \text {. }
$$

Diese Formel bleibt richtig, so lange man um den Punkt $x y z$ Kugelflächen legen kann, in welchen die Function $\Phi$ die oben genannten Stetigkeitsbedingungen nicht verletzt. Nehmen wir nun an, die Function $\Phi$ sei so beschaffen, dass sie im ganzen unendlichen Raum die Stetigkeitsbedingungen erfüllt, dass also der Radius jener Kugel ins Unendliche wachsen kann, so können wir die Formel (4.) zur Bestimmung des folgenden Integrals anwenden:

$$
\text { (5.) } \int_{-\infty}^{+\infty} \int_{-\infty}^{+\infty} \int_{-\infty}^{+\infty} \Phi(a b c) e^{-p^{2}\left[(a-x)^{3}+(b-y)^{3}+(c-z)^{3}\right]} d a d b d c=\Omega \text {. }
$$

Führt man in diesem Integral Polarcoordinaten ein, was, so lange $p$ reell und von 0 verschieden ist (oder wenn $p^{2}$ imaginär sein sollte, so lange sein reeller Theil positiv ist), unbedingt erlaubt ist, indem man setzt:

$$
\begin{aligned}
& a-x=r \sin \vartheta \sin \varphi, \\
& b-y=r \sin \vartheta \cos \varphi, \quad \cos \vartheta=\mu, \\
& c-z=r \cos \vartheta, \quad
\end{aligned}
$$


so ergiebt sich:

$$
\Omega=\int_{0}^{\infty} r^{2} e^{-p^{2} r^{2}} d r \int_{-1}^{+1} \int_{-\pi}^{+\pi} \Phi d i d \varphi
$$

und durch Anwendung der Formel (4.):

$$
\Omega=\frac{4 \pi}{m} \Phi(x y z) \int_{0}^{\infty} r e^{-p^{2} r^{2}} \sin m r d r .
$$

Nach einer bekannten Formel ist:

$$
\int_{10}^{\infty} r e^{-p^{2} r^{2}} \sin m r d r=\frac{m}{4 p^{3}} \sqrt{ } / \pi e^{-\frac{m^{2}}{4 p^{9}}}
$$

und demnach:

$$
\text { (6.) } \quad \int_{-\infty}^{+\infty} \int_{-\infty}^{+\infty} \int_{-\infty}^{+\infty} \Phi(a b c) e^{-p^{2}\left[(a-x)^{3}+(b-y)^{2}+(c-z)^{2}\right]} d a d b d c=\frac{\pi^{\frac{3}{2}}}{p^{3}} e^{-\frac{m^{3}}{4 p^{3}}} \boldsymbol{\Phi}(x y z) \text {. }
$$

Dies ist die in der Einleitung erwähnte Formel. Man bemerkt aber sofort, dass sich diese Formel noch bedeutend verallgemeinern lässt.

Es lässt sich nämlich genau dasselbe Verfahren anwenden auf das Integral :

$$
\int_{-\infty}^{+\infty} \int_{-\infty}^{+\infty} \int_{-\infty}^{+\infty} \boldsymbol{\Phi}(\boldsymbol{a b c}) \varphi\left(\sqrt{(\boldsymbol{x}-\boldsymbol{a})^{2}+(y-b)^{2}+(z-c)^{2}}\right) d a d b d c,
$$

worin $\varphi$ eine beliebige Function bedeutet, welche an die einzige Bedingung geknüpft ist, dass das vorstehende Integral nicht nur convergent ist, sondern auch seinen Werth nicht ändert durch Einführung neuer Variabeln und beliebige Anordnung der Integrationsfolge. Unter dieser Voraussetzung gelangt man auf demselben Wege wie oben zu der Formel:

$$
\left\{\begin{array}{c}
\int_{-\infty}^{+\infty} \int_{-\infty}^{+\infty} \int_{-\infty}^{+\infty} \boldsymbol{\Phi}(a b c) \varphi\left\{\sqrt{(\boldsymbol{x}-a)^{2}+(y-b)^{2}+(z-c)^{2}}\right\} d a d b d c \\
=\frac{4 \pi}{m} \Phi(x y z) \int_{y}^{\infty} r \varphi(r) \sin m r d r
\end{array}\right.
$$

Macht man z. B. über die Function $\varphi$ die Annahme:

$$
\varphi(\boldsymbol{r})=\frac{\boldsymbol{e}^{-\varepsilon r}}{\boldsymbol{r}^{p}},
$$

so lässt sich das Integral auf der rechten Seite ausführen. Es ist nämlich:

$$
\int_{0}^{\infty} \dot{r}^{1-p} e^{-\varepsilon r} \sin m r d r
$$


der imaginäre Theil des Integrals:

worin :

$$
\int_{0}^{\infty} r^{1-p} e^{-(\varepsilon-i m) r} d r=\frac{\Gamma(2-p)}{(\varepsilon-i m)^{2-p}}
$$

$$
(\varepsilon-i m)^{2-p}=\left(\varepsilon^{2}+m^{2}\right)^{\frac{1}{2}(2-p)} e^{-i(2-p) \operatorname{arc~t} \frac{m}{\varepsilon}}
$$

und $\operatorname{arctg}$ zwischen $-\frac{1}{2} \pi$ und $+\frac{1}{2} \pi$ zu nehmen ist; und demnach:

$$
\begin{aligned}
\int_{1}^{\infty} r^{1-p} e^{-\varepsilon r} \sin m r d r & =-\Gamma(2-p) \cdot\left(\varepsilon^{2}+m^{2}\right)^{\frac{1}{2}(p-2)} \sin (p-2) \operatorname{arctg} \frac{m}{\varepsilon} \\
& =+\frac{\pi}{\Gamma(p-1)}\left(\varepsilon^{2}+m^{2}\right)^{\frac{1}{2}(p-2)} \frac{\sin (p-2) \operatorname{arctg} \frac{m}{\varepsilon}}{\sin p \pi},
\end{aligned}
$$

welche letztere Form gültig ist, so lange $p$ zwischen 1 und 3 liegt, und auch dann noch besteht, wenn die bisher positiv vorausgesetzte Grösse $\varepsilon$ in 0 übergeht. Demnach erhält man folgende Formel:

$$
\left\{\begin{array}{l}
\int_{-\infty}^{+\infty} \int_{-\infty}^{+\infty} \int_{-\infty}^{+\infty} d a d b d c \Phi(a b c) \frac{e^{-\varepsilon \sqrt{(u-x)^{3}+(b-y)^{2}+(c-z)^{2}}}}{\left((a-x)^{2}+(b-y)^{2}+(c-z)^{2}\right)^{\frac{1}{2} p}} \\
=\frac{4 \pi^{2}}{m} \frac{\Phi(x y z)}{\Gamma(p-1)}\left(\varepsilon^{2}+m^{2}\right)^{\frac{1}{2}(p-2)} \frac{\sin (p-2) \operatorname{arctg} \frac{m}{\varepsilon}}{\sin p \pi} .
\end{array}\right.
$$

Ist die Function $\Phi$ und der Exponent $p$ so beschaffen, dass das dreifache Integral auf der linken Seite bis $\varepsilon=0$ inclusive eine stetige Function von $\varepsilon$ ist, so kann in der vorstehenden Formel $\varepsilon=0$ gesetzt werden, und es ergiebt sich :

$$
\text { (9.) } \int_{-\infty}^{+\infty} \int_{-\infty}^{+\infty} \int_{-\infty}^{+\infty} \frac{d a d b d c \Phi(a b c)}{\left\{(x-a)^{2}+(y-b)^{2}+(z-c)^{2}\right\}^{\frac{1}{3} p}}=-\frac{2 \pi^{2} m^{p-3}}{\Gamma(p-1) \cos \frac{1}{2} p \pi} \boldsymbol{\Phi}(x y z) \text {. }
$$

\$. 2 .

Ich mache zunächst eine Anwendung der Formel (6.). Nimmt man an, es sei $\Phi(a b c)=\Phi(a b)$ von $c$ unabhängig, so geht dieselbe über in :

$$
\text { (10.) } \quad \int_{-\infty}^{+\infty} \int_{-\infty}^{+\infty} \Phi(a b) e^{-p^{2}\left[(x-a)^{2}+(y-b)^{2}\right]} d a d b=\frac{\pi}{p^{2}} e^{-\frac{m^{2}}{4 p^{2}}} \Phi(x y) \text {. }
$$

Ein specieller Fall der Function $\Phi$, welche allen hier gestellten Anforderungen genügt und gleichzeitig nur von $a^{2}+b^{2}=\varrho^{2}$ abhängt, ist die Besselsche Function 0ter Ordnung:

$$
\Phi=J^{(1)}(m \varphi),
$$


welche der Differentialgleichung genügt:

$$
-m^{2} \Phi=\frac{\partial^{2} \Phi}{\partial \varrho^{2}}+\frac{1}{\varrho} \frac{\partial \Phi}{\partial \varrho},
$$

und welche ausgedrückt werden kann durch die stets convergente Reihe:

$$
J(z)=1-\frac{z^{2}}{2^{2}}+\frac{z^{4}}{2^{2} \cdot 4^{2}}-\cdots
$$

oder durch das Integral:

$$
J(z)=\frac{1}{\pi} \int_{0}^{\pi} \cos (z \sin \omega) d \omega .
$$

Setzt man diese Function in die Formel (10.) ein, setzt in derselben $x=0$, $y=0$ und transformirt das Integral auf Polarcoordinaten, so erhält man die Formel:

$$
\text { (11.) } \int_{0}^{\infty} \varrho e^{-p^{2} \varrho^{2}} J^{(1)}(m \varrho) d \varrho=\frac{1}{2 p^{2}} e^{-\frac{m^{2}}{4 p^{2}}} \text {. }
$$

Dieses bestimmte Integral scheint um so bemerkenswerther zu sein, als das demselben entsprechende Integral für die trigonometrischen Functionen nicht in endlicher Form dargestellt werden kann. Insofern nämlich $\varrho J^{0}(m \varrho)$ eine ungerade Function ist, würde unserm Integral entsprechen: $\int_{0}^{\infty} e^{-p^{2} \rho^{2}} \sin m \rho d \varrho$, was auf eine unvollständige $I$-Function zurückkommt.

Um dieses Integral zu verallgemeinern, geben wir zunächst die Voraussetzung auf, dass $\Phi(a b)$ allein von $\varrho$ abhängig sei. Setzt man, um Polarcoordinaten einzuführen:

$$
\begin{array}{ll}
a=\rho \cos \varphi, & x=r \cos q, \\
b=\rho \sin \varphi, & y=r \sin q,
\end{array}
$$

so muss $\Phi$ der Differentialgleichung genügen:

$$
-m^{2} \boldsymbol{\Phi}=\frac{\partial^{2} \Phi}{\partial \varrho^{2}}+\frac{1}{\varrho} \frac{\partial \Phi}{\partial \varrho}+\frac{1}{\varrho^{2}} \frac{\partial^{2} \Phi}{\partial \varphi^{2}} .
$$

Nimmt man an, es sei:

$$
\Phi=\Psi(A \cos h \varphi+B \sin h \varphi),
$$

wo $\Psi$ nun nur noch von $\varrho$ abhängig ist, und $h$ der Eindeutigkeit wegen eine ganze Zahl bedeuten muss, so erhält man für $\Psi$ die Differentialgleichung:

$$
\left(\frac{h^{2}}{\varrho^{2}}-m^{2}\right) \Psi=\frac{\partial^{2} \Psi}{\partial \varrho^{2}}+\frac{1}{\varrho} \frac{\partial \Psi}{\partial \varrho} \text {. }
$$

Diese Gleichung hat eine durchaus stetige Lösung, nämlich die Besselsche Function $h^{\text {ter }}$ Ordnung:

$$
\Psi=J^{(h)}(m \varrho)
$$


wo $J^{(h)}(z)$ wieder durch eine immer convergente Reihe und durch ein bestimmtes Integral ausgedrückt werden kann:

$$
\begin{aligned}
J^{(h)}(z) & =\frac{z^{4}}{2.4 \ldots 2 h}\left\{1-\frac{z^{2}}{2.2 h+2}+\frac{z^{4}}{2.4 .2 h+2.2 h+4}-\cdots\right\} \\
& =\frac{1}{\pi} \int_{0}^{\pi} \cos (z \sin \varphi-h \varphi) d \varphi=\frac{z^{h}}{1.3 .5 \ldots 2 h-1} \cdot \frac{1}{\pi} \int_{0}^{\pi} \cos (z \cos \varphi) \sin ^{2 h} \varphi d \varphi .
\end{aligned}
$$

Setzt man diese Function in die Gleichung (10.) ein, nachdem man das Integral auf Polarcoordinaten transformirt hat, so ergiebt sich:

$$
\begin{gathered}
e^{-p^{2} r^{2}} \int_{0}^{\infty} \rho e^{-p^{2} \varphi^{2}} J^{(h)}(m \varrho) d \varrho \int_{-\pi}^{+\pi} e^{2 p^{2} r \varphi \cos (\varphi-q)}(A \cos h \varphi+B \sin h \varphi) d \varphi \\
=\frac{\pi}{p^{2}} e^{-\frac{m^{3}}{4 p^{2}}} J^{(h)}(m r)(A \cos h q+B \sin h q) .
\end{gathered}
$$

In dieser Formel setze man:

$$
A=1, \quad B=i, \quad q=\frac{1}{2} \pi .
$$

Dann nimmt das Integral in Bezug auf $\varphi$ folgende Gestalt an:

$$
\int_{-\pi}^{+\pi} e^{2 p^{2} r \rho \sin \varphi+i \hbar \varphi} d \varphi
$$

welches sich leicht auf folgende Form bringen lässt:

$\int_{1}^{\pi}\left\{e^{2 p^{2} r \rho \sin \varphi+i h \varphi}+e^{-2 p^{3} r \rho \sin \varphi-i h \varphi}\right\} d \varphi=2 \int_{0}^{\pi} \cos \left(2 i p^{2} r \varphi \sin \varphi-h \varphi\right) d \varphi=2 \pi J^{(h)}\left(2 i p^{2} r \varphi\right)$, und darnach wird das obige Integral:

$$
\int_{0}^{\infty} \varrho e^{-p^{2} \rho^{2}} J^{(h)}(m \varrho) J^{(h)}\left(2 i p^{2} r \varrho\right) d \varrho=\frac{i^{h}}{2 p^{2}} e^{-\frac{m^{2}}{4 p^{2}}+p^{2} r^{2}} J^{(h)}(m r)
$$

oder in etwas mehr symmetrischer Form geschrieben, indem man $2 p^{2} r=n$ setzt:

$$
\int_{0}^{\infty} \varrho e^{-p^{2} \rho^{2}} J^{(h)}(m \varrho) J^{(h)}(i n \varrho) d \varrho=\frac{i^{h}}{2 p^{2}} e^{-\frac{m^{3}-n^{2}}{4 p^{2}}} J^{(h)}\left(\frac{m n}{2 p^{2}}\right) \text {. }
$$

Lässt man in dieser Formel $n$ gegen 0 abnehmen und setzt für die Functionen $J^{(h)}(i n \varrho), J^{(h)}\left(\frac{n m}{2 p^{2}}\right)$ die ersten Glieder der Reihenentwicklungen, so erhält man die Verallgemeinerung der Formel (11.):

$$
\int_{1}^{\infty} \varrho^{h+1} e^{-y^{2} \rho^{2}} J^{(h)}(m \varrho) d \rho=\frac{m^{h}}{\left(2 p^{2}\right)^{h+1}} e^{-\frac{m^{2}}{4 p^{2}}} .
$$

Alle unsere Betrachtungen bleiben für diese Integrale auch dann noch richtig, wenn man $m$ irgendwie complex, also beispielsweise auch rein ima- 
ginär annimmt. Die Functionen $J^{(h)}$ werden in diesem Falle allerdings unendlich für unendliche Werthe des Arguments, jedoch so, dass die Convergenz der Integrale in keiner Weise beeinträchtigt wird, wegen des Factors $e^{-p^{2} \varrho^{2}}$. Denn für sehr grosse Werthe der Variablen nähern sich die Functionen $J^{(h)}(m \varrho)$ dem Grenzwerth $\frac{A \cos m \varrho+B \sin m \varrho}{\sqrt{l}}$.

Ebenso kann in der Formel (12.) $n$ irgendwie complex angenommen werden, denn sowohl rechts als links stehen durchaus stetige Functionen von $n$, welche nur für unendliche Werthe von $n$ unendlich werden, und die Werthe beider Functionen stimmen längs einer Linie, nämlich der ganzen Linie, auf der $n$ reell ist, mit einander überein. Solche Functionen müssen aber überhaupt übereinstimmen *).

Setzt man also - in an die Stelle von $n$, so nimmt (12.) die Form an:

$$
\text { (14.) } \int_{0}^{\infty} \varrho e^{-p^{2} \varrho^{2}} J^{(h)}(m \varrho) J^{(h)}(n \varrho) d \varrho=\frac{e^{-\frac{m^{2}+n^{2}}{4 p^{2}}}}{2 p^{2}} i^{h} J^{(h)}\left(\frac{m n}{2 i p^{2}}\right) \text {. }
$$

In ganz ähnlicher Weise lässt sich auch das allgemeinere Integral finden:

$$
\int_{0}^{\infty} \dot{\varphi}^{2} e^{-p^{2} \rho^{2} J^{(1)}}(m \varrho) d \varrho \text {, }
$$

worin $\lambda$ eine ungerade positive ganze Zahl bedeutet. Hat man dieses Integral gefunden, dann ergeben sich mit Hülfe der recurrenten Formel für die Besselschen Functionen:

$$
\left.J^{(h+1)}(\boldsymbol{z})=\frac{h}{z} J^{(h)}(z)-\frac{\partial J^{(h)}(z)}{\partial z}{ }^{* *}\right)
$$

die Integrale von der Form:

$$
\int_{0}^{\infty} \rho^{h+\lambda} e^{-p^{2} \varphi^{2}} J^{(h)}(m \varphi) d \varphi,
$$

wo $\lambda$ wieder eine ungerade ganze $Z$ ahl bedeutet, welche bis $-2 h+1$ abnehmen kann. Da aber diese allgemeinen Formeln keine einfache Gestalt annehmen, und daher auch ohne besonderes Interesse sein dürften, so übergehe ich diese Ableitung und will nur als Beispiel dieser Art das eine Integral anführen:

$$
\int_{0}^{\infty} e^{-p^{2} \varrho^{2}} J^{(1)}(m \varrho) d \varrho=\frac{1}{m}\left(1-e^{-\frac{m^{9}}{4 p^{2}}}\right)
$$

*) Vgl. Riemann: Grundl. der Theorie der Functionen einer veränderl. complexen Grösse, §. 15 .

**) Vgl. C. Neumann: Theorie der Besselschen Functionen. Leipzig 1867 p. 22. Journal für Mathematik Bd. LXIX. Heft 3. 
welches sich mit Hülfe der angeführten Recursionsformel aus dem bereits gefundenen Integrale sofort ergiebt.

\section{S. 3.}

Man kann nun in ähnlicher Weise wie bisher von der Gleichung (6.), so von der Gleichung (9.) $\$$. 1 ausgehen, um zur Bestimmung des Integrals:

$$
\int_{0}^{\infty} \dot{r}^{q-h-1} J^{(h)}(\boldsymbol{m r} r) d r
$$

zu gelangen. Dieser Weg ist aber etwas mühselig, und eine strenge Rechtfertigung des Verfahrens, wenigstens für das ganze Intervall der Gültigkeit der Endformel nicht ohne Schwierigkeiten. Ich werde daher ein anderes Verfahren einschlagen, indem ich die im vorigen $\$$. gefundenen Resultate benutze, wobei man nicht nur am schnellsten zu der Endformel in ihrer einfachsten Gestalt gelangt, sondern auch der Zulässigkeit der auszuführenden Operationen vollständig versichert ist. Es handelt sich also um die Bestimmung des Integrals :

$$
\text { (1.) } \int_{0}^{\infty} r^{q-h-1} J^{(h)}(m r) d r \text {, }
$$

welches convergent bleibt, so lange:

$$
0<q<h+\frac{3}{2}
$$

wie man erkennt, wenn man die bekannten Grenzwerthe der Functionen $J^{(h)}$ berücksichtigt. In diesem Integral setzt man nun einer vielfach benutzten Eulerschen Formel zu Folge:

und erhält demnach :

$$
\left.\frac{1}{r^{2 h+2-q}}=\frac{1}{\Gamma(h+1}-\frac{1}{2} q\right) \int_{0}^{\infty} s^{h-\frac{1}{2} q} e^{-r^{3} s} d s
$$

$$
\int_{0}^{\infty} r^{q-h-1} J^{(h)}(m r) d r=\frac{1}{\Gamma\left(h+1-\frac{1}{2} q\right)} \int_{0}^{\infty} d r \int_{0}^{\infty} s^{h-\frac{1}{2} q} r^{h+1} J^{(h)}(m r) e^{-r^{3 s}} d s .
$$

Die weitere Entwicklung der Formel beruht nun auf der Umkehrung der Integrationsfolge des Doppelintegrals auf der rechten Seite der Gleichung (2.). Dass diese Umkehrung gestattet ist, ist zwar nicht von selbst klar, da das Integral nicht mehr convergent ist, wenn man für die Function unter dem Integralzeichen durchaus ihre absoluten Werthe setzt. Es lässt sich aber in diesem Fall leicht nachweisen, dass die Umkehrung zu richtigen Resultaten führen muss. Am einfachsten geschieht dies wohl auf folgende Weise:

Erstreckt man die Integration in Bezug auf $s$ statt von 0 von einer positiven Grösse $\varepsilon$ aus, so wird das Integral, wie man leicht einsieht, in ein 
anderes verwandelt, welches, so lange $\varepsilon$ von 0 merklich verschieden ist, noch convergent bleibt, wenn für die Function unter dem Zeichen die absoluten Werthe gesetzt werden, welches demnach die Umkehrung ohne Weiteres gestattet. Wenn nun die Integrale, welche man auf diese Weise erhält, für gehörig kleine Werthe von $\varepsilon$ von den entsprechenden Integralen, in denen geradezu $\varepsilon=0$ gesetzt ist, und zwar sowohl in der Weise genommen, wie die Formel (2.) es verlangt, als auch nachdem die Integrationsfolge umgekehrt worden ist, beliebig wenig unterschieden sind, so ist die Umkehrung der Integrationsfolge in dem Doppelintegral der Formel (2.) gestattet. Diese Stetigkeit der Integrale in Bezug auf $\varepsilon$ ist aber für das durch die Umkehrung gewonnene Integral unmittelbar einzusehen, und auch für das in der Formel (2.) vorkommende Integral ist sie leicht nachzuweisen *).

Demnach geht nun die Gleichung (2.) in folgende über:

$$
\int_{0}^{\infty} r^{q-h-1} J^{(h)}(m r) d r=\frac{1}{\Gamma\left(h+1-\frac{1}{2} q\right)} \int_{0}^{\infty} \dot{s}^{h-\frac{1}{2} q} d s \int_{0}^{\infty} e^{-r^{3} s} r^{h+1} J^{(h)}(m r) d r .
$$

Die Integration in Bezug auf $r$ lässt sich hier nun mittelst der Formel (13.) des vorigen Paragraphen ausführen, wodurch man erhält:

$$
\int_{0}^{\infty} r^{q-h-1} J^{(h)}(m r) d r=\frac{m^{h}}{2^{h+1} \Gamma\left(h+1-\frac{1}{2} q\right)} \int_{0}^{\infty} e^{-\frac{m^{2}}{4 s}} s^{-\frac{1}{2} q-1} d s .
$$

Das nach $s$ genommene Integral auf der rechten Seite geht aber durch die Substitution $z=\frac{1}{s}$ in eine $l$-Function über und ergiebt so die merkwürdig einfache Formel:

$$
\int_{0}^{\infty} r^{q-h-1} J^{(h)}(m r) d r=m^{h-q} 2^{q-h-1} \frac{\Gamma\left(\frac{1}{2} q\right)}{\Gamma\left(h+1-\frac{1}{2} q\right)} .
$$

Diese Formel stimmt für den Fall $h=0$ mit der von Herrn Lipschitz ge-

*) Am einfachsten führt zu diesem Beweis ein Satz, den ich einer Mittheilung des Herrn Paul du Bois-Reymond verdanke, und der für unseren Fall etwas specialisirt, so lautet: Sind die beiden Integrale

$$
\int_{R}^{\infty} f(r) \varphi(r) d r, \quad \int_{R}^{\infty} f(r) d r
$$

convergent, und nimmt $\varphi(r)$ zwischen den Grenzen beständig ab oder beständig zu, indem es sich der Grenze 0 nähert, so ist:

$$
\int_{R}^{\infty} f(r) \varphi(r) d r=\varphi(R) \int_{R}^{\rho} f(r) d r
$$

wenn $\varrho$ eine nicht näher bekannte, zwischen $\boldsymbol{R}$ und $\infty$ gelegene Grösse bedeutet. 
fundenen überein, wie man mit Hülfe der bekannten Gaussischen Relation zwischen den $T$-Functionen erkennt.

Ich hebe noch zwei bemerkenswerthe specielle Fälle dieser Formel hervor: den ersten derselben erhält man durch die Annahme $q=h+1$, welche immer gestattet ist. Diese Annahme ergiebt:

$$
\text { (4.) } \int_{0}^{\infty} J^{(h)}(m r) d r=\frac{1}{m} \text {. }
$$

Dieses Integral hat also für die Besselschen Functionen aller Ordnungen denselben Werth.

Die zweite specielle Annahme ist $h=q$, welche nur in dem Fall $h=0$ nicht gestattet ist, und führt zu dem Integral:

$$
\int_{0}^{\infty} \frac{J^{(h)}(m r) d r}{r}=\frac{1}{h} \text {. }
$$

Dieses Integral ist also von $m$ unabhängig, was auch unmillelbar einzusehen ist. Als Function von $m$ betrachtet ist dasselbe aber discontinuirlich, denn für $m=0$ hat es den Werth 0 . Ist $h$ eine ungerade Zahl, so hat das Integral $\mathrm{zu}$ beiden Seiten der Unstetigkeitsstelle entgegengesetzte Werthe; ist aber $h$ eine gerade Zahl, so sind die Werthe zu beiden Seiten der Unstetigkeitsstelle dieselben, so dass man hier eine Function hat, welche für alle Werthe von $m$ constant ist, nur für $m=0$ einen isolirten Werth besitzt.

\section{S. 4.}

Genau dasselbe Verfahren, was bisher auf die Besselschen Functionen angewandt wurde, führt zu ganz entsprechenden Resultaten bei einer anderen Classe von Functionen, welche in der Theorie der Bewegung der Wärme in einer Kugel eine wichtige Rolle spielen, und die mit den Besselschen Functionen die grösste Verwandtschaft haben, dergestalt, dass man sie geradezu bezeichnen kann mit $J^{\left(\frac{2 h+1}{2}\right)}(z)$. Die in Rede stehenden Functionen gehen nämlich aus den Besselschen Functionen hervor, insofern dieselben durch die Differentialgleichung definirt sind, dadurch, dass man die Ordnungszahl $h$ in ein ungerades Vielfache von $\frac{1}{2}$ verwandelt, und auch die unendliche Reihe und das bestimmte Integral

$$
J^{(h)}(z)=\frac{z^{h}}{1.3 .5 \ldots(2 h-1)} \frac{1}{\pi} \int_{0}^{\pi} \sin ^{2 h} \omega \cos (z \cos \omega) d \omega
$$


lassen sich ohne Weiteres auf diese Functionen übertragen, wenn man eine kleine, natürlich sich bietende Modification mit dem numerischen Factor, der vor dem Ganzen steht, vornimmt. Das Integral, welches die Besselsche Function zuerst definirte, wird allerdings durch ein etwas anders gebildetes, aber nicht minder einfaches Integral ersetzt.

Um zu diesen Functionen zu gelangen, gehe ich aus von der auf räumliche Polarcoordinaten transformirten Gleichung (1.) \$. 1 und nehme der Einfachheit halber an, die Lösung $\Phi$ dieser Gleichung sei unabhängig von dem Winkel $\varphi$, wodurch diese Gleichung die Gestalt annimmt:

$$
\text { (1.) }-\boldsymbol{m}^{2} \boldsymbol{\Phi}=\frac{1}{r^{2}}\left\{\frac{\partial}{\partial \boldsymbol{r}}\left(\boldsymbol{r}^{2} \frac{\partial \boldsymbol{\Phi}}{\partial \boldsymbol{r}}\right)+\frac{\partial}{\partial \mu}\left(1-\mu^{2}\right) \frac{\partial \Phi}{\partial \mu}\right\} \text {. }
$$

Nimmt man weiter an, $\Phi$ habe die Form:

$$
\boldsymbol{\Phi}=\boldsymbol{R}^{(h)} \boldsymbol{P}^{\left(l_{1}\right)}(\boldsymbol{\mu}),
$$

wo $P^{(h)}$ die einfache Kugelfunction hter Ordnung, also eine für alle Werthe von $\vartheta=\operatorname{arc} \cos \mu$ stetige und eindeutige Function, $R^{(h)}$ eine Function von $r$ allein bedeutet, so ergiebt sich für $R$ die Differentialgleichung:

$$
\frac{\partial^{2} R^{(h)} \cdot r}{\partial r^{2}}+\left(m^{2}-\frac{h \cdot h+1}{r^{2}}\right) r R^{(h)}=0 .
$$

Definirt man also eine Function $S^{(h)}(r)$ durch die Gleichung:

$$
\text { (2.) } \frac{\partial^{2} S^{(h)}}{\partial r^{2}}+\left(1-\frac{h \cdot h+1}{r^{2}}\right) S^{(h)}=0
$$

und durch die Bedingung, dass die Function $S^{(h)}$ durchaus endlich und stetig und für $r=0$ mindestens wie die erste Potenz von $r$ verschwinden soll, wodurch $S$ bis auf einen constanten Factor bestimmt ist, so erhält man

$$
R^{(h)}=A \cdot \frac{S^{(h)}(m r)}{r}
$$

und hat damit eine Lösung der Gleichung (1.), welche den Bedingungen des am Anfang aufgestellten Satzes genügt. Setzt man noch

$$
\text { (3.) } \quad \boldsymbol{S}^{(h)}(\boldsymbol{r})=\sqrt{ } \boldsymbol{r} \boldsymbol{J}^{\left(\frac{2 h+1}{2}\right)}(\boldsymbol{r}),
$$

so erhält man für die Function $J$ die Differentialgleichung:

$$
\text { (4.) } \frac{\partial^{2} J\left(\frac{2 h+1}{2}\right)}{\partial r^{2}}+\frac{1}{r} \frac{\partial J^{\left(\frac{2 h+1}{2}\right)}}{\partial r}+\left(1-\frac{\left(\frac{2 h+1}{2}\right)^{2}}{r^{2}}\right) J^{\left(\frac{2 h+1}{2}\right)}=0 \text {, }
$$

welche mit der Differentialgleichung der Besselschen Functionen übereinstimmt. 
Für die Function $S$ lassen sich nun verschiedene Ausdrücke aufstellen, welche sich durch Substitution in die Differentialgleichung leicht verificiren lassen: Man erhält, wenn man den constanten Factor in den Functionen $S$ in geeigneter Weise bestimmt:

$$
\begin{aligned}
& S^{(h)}(r)=\frac{r}{2 i^{h}} \int_{-1}^{+1} P^{(h)}(\mu) e^{i r \mu} d \mu, \\
& \left.S^{(h)}(r)=\frac{r^{h+1}}{2.4 .6 \ldots 2 h} \int_{0}^{\frac{1}{3} \pi} \sin ^{2 h+1} \omega \cos (r \cos \omega) d \omega^{*}\right) .
\end{aligned}
$$

Die Uebereinstimmung der beiden Ausdrücke lässt sich leicht durch eine partielle Integration nachweisen, wenn man für die Kugelfunction $\boldsymbol{P}$ den bekannten Ausdruck durch einen $h$ fachen Differentialquotienten einsetzt. Ferner ergiebt sich aus jedem der beiden Ausdrücke (5.), (6.) für $S$ die stets convergente Entwicklung:

$$
S^{(h)}(r)=\frac{r^{h+1}}{1.3 .5 \ldots 2 h+1}\left\{1-\frac{r^{2}}{2.2 h+3}+\frac{r^{4}}{2.4 .2 h+3.2 h+5}-\cdots\right\} .
$$

Man sieht, dass sowohl das Integral (6.) als die Reihe (7.), wenn man dieselben durch $\bigvee / r$ dividirt, aus den entsprechenden Ausdrücken für $J^{(h)}(r)$ hervorgehen durch Vertauschung von $h$ mit $\frac{2 h+1}{2}$, abgesehen von dem etwas veränderten constanten Factor.

Ausserdem lassen sich die Functionen $\boldsymbol{S}^{(h)}(\boldsymbol{r})$ noch durch geschlossene Reihen ausdrücken, auf die es mir hier aber nicht weiter ankommt, nur bemerke ich noch, dass zwischen den Functionen $S^{(h)}$ oder $J^{\left(\frac{2 h+1}{2}\right)}$ genau dieselbe Recursionsformel besteht, wie zwischen den Besselschen Functionen mit ganzzahligem Index, nämlich:

$$
\begin{aligned}
\frac{2 h+1}{r} S^{(h)}(r) & =S^{(h-1)}(r)+S^{(h+1)}(r), \\
\frac{2 h+1}{r} J^{\left(\frac{2 h+1}{2}\right)}(r) & =J^{\left(\frac{2 h-1}{2}\right)}(r)+J^{\left(\frac{2 h+3}{2}\right)}(r),
\end{aligned}
$$

welche sich aus jedem der oben gegebenen Ausdrücke für diese Functionen leicht herleiten lassen. Demnach können die Functionen $S$ alle linear ausgedrückt werden durch die beiden ersten derselben:

$$
S^{(1)}=\sin r, \quad S^{(1)}=\frac{\sin r}{r}-\cos r .
$$

*) Diese Ausdrücke für die Function $\boldsymbol{S}$ sind mir bekannt aus einer Vorlesung des Herrn Prof. Neumann zu Königsberg. 


\section{\$. 5 .}

Ich benutze nun ganz wie oben die Formel (6.) \$. 1 zur Ableitung gewisser bestimmter Integrale, die von den Functionen $J^{\left(\frac{2 h+1}{2}\right)}(r)$ abhängen. $\mathrm{Zu}$ dem Ende mache ich in der Formel (6.) die Annahme:

$$
\begin{gathered}
x=y=0, \\
\Phi(a b c)=\frac{1}{\sqrt{ } r} J^{\left(\frac{2 h+1}{2}\right)}(m r) P^{(h)}(\mu), \\
\Phi(x y z)=\frac{1}{\sqrt{ } z} J^{\left(\frac{2 h+1}{2}\right)}(m z),
\end{gathered}
$$

wonach durch Einführung von Polarcoordinaten unter dem Integralzeichen die Formel (6.) die Gestalt annimmt:

$$
\left\{\begin{array}{c}
2 \pi e^{-p^{2} z^{2}} \int_{0}^{\infty} r^{\frac{3}{2}} e^{-p^{2,2}} J^{\left(\frac{2 h+1}{2}\right)}(m r) d r \int_{-1}^{+1} P^{(h)}(u) e^{2 p^{2} r z \mu} d u \\
=\frac{\pi^{\frac{3}{2}}}{p^{3}} e^{-\frac{m^{2}}{4 p^{3}}} \cdot \frac{1}{\sqrt{z}} J^{\left(\frac{2 h+1}{2}\right)}(m z) .
\end{array}\right.
$$

Die Integration in Bezug auf $\mu$ lässt sich hier mittelst der Formel (5.) ausführen, und man erhält, wenn man:

setzt:

$$
2 p^{2} z=n
$$

$$
\text { (9.) } \int_{1}^{\infty} r e^{-p^{2} r^{2}} J^{\left(\frac{2 h+1}{2}\right)}(m r) J^{\left(\frac{2 h+1}{2}\right)}(-i n r) d r=\frac{\sqrt{2 \pi}(-i)^{\frac{2 h+1}{2}}}{4 p^{2}} e^{-\frac{m^{2}-n^{3}}{4 p^{3}}} J^{\left(\frac{2 h+1}{2}\right)}\left(\frac{m n}{2 p^{2}}\right) \text {, }
$$

worin wieder wie oben $m$ und $n$ nicht nothwendig reell zu sein brauchen. Lässt man nun hierin $n$ gegen 0 abnehmen, indem man für die beiden von $n$ abhängigen Functionen $J$ die ersten Glieder der Reihenentwicklung setzt, so erhält man die der Formel (13.) $\$$. 2 entsprechende Formel:

$$
\text { (10.) } \int_{0}^{\infty} r^{\frac{2 h+3}{2}} e^{-p^{2} r^{2}} J^{\left(\frac{2 h+1}{2}\right)}(m r) d r=\frac{\sqrt{\frac{1}{2} \pi m^{\frac{2 h+1}{2}}}}{\left(2 p^{2}\right)^{\frac{2 h+3}{2}}} e^{-\frac{m^{2}}{4 p^{2}}} \text {, }
$$

welche bis auf den Factor $\sqrt{\frac{1}{2} \pi}$ mit jener übereinstimmt. Für die beiden Fälle $h=0$ und $h=1$ lässt sich diese Formel leicht mit Hülfe der bekannten Integrale verificiren.

Es soll nun in ähnlicher Weise wie in $\$$. 3 das Integral

$$
\text { (11.) } \int_{0}^{\infty} r^{q-\frac{2 h+3}{2}} J^{\frac{2 h+1}{2}}(m r) d r
$$


aus dem soeben gefundenen abgeleitet werden. Das Integral (11.) behält einen Sinn, so lange

$$
0<q<h+2
$$

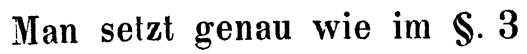

$$
\frac{1}{r^{2 h+3-q}}=\frac{1}{\Gamma\left(\frac{2 h+3-q}{2}\right)} \int_{i}^{\infty} s^{\frac{2 h+1-q}{2}} e^{-r^{2} s} d s
$$

und verfährt ganz in derselben Weise wie dort, indem man von der Formel (10.) Gebrauch macht, und gelangt so zu dem Resultat:

$$
\text { (12.) } \int_{0}^{\infty} r^{q-\frac{2 h+3}{2}} J^{\frac{2 h+1}{2}}(m r) d r=\sqrt{\frac{1}{2} \pi} m^{\frac{2 h+1}{2}-q} 2^{q-\frac{2 h+3}{2}} \frac{\Gamma\left(\frac{1}{2} q\right)}{\Gamma\left(\frac{2 h+3-q}{2}\right)} \text {, }
$$

was wieder, abgesehen von dem Factor, $\sqrt{\sqrt{2} \pi}$ mit der Gleichung (3.) $\$$. 3 übereinstimmt.

Macht man wieder dieselben speciellen Annahmen wie oben, indem man $q=\frac{2 h+3}{2}$ und $q=\frac{2 h+1}{2}$ setzt, so erhält man die beiden Integrale:

$$
\begin{aligned}
& \text { (13.) } \int_{0}^{\infty} J^{\left(\frac{2 h+1}{2}\right)}(m r) d r=\sqrt{\frac{1}{2} \pi} \cdot \frac{1}{m}, \\
& \text { (14.) } \int_{0}^{\infty} J^{\left(\frac{2 h+1}{2}\right)}(m r) \frac{d r}{r}=\sqrt{\frac{1}{2} \pi} \cdot \frac{2}{2 h+1} .
\end{aligned}
$$

Diese Formeln sind nur dann völlig unzweifelhaft, wenn $m$ reell und positiv ist. Soll $m$ auch imaginär sein, so muss der reelle Theil von $m^{2}$ positiv sein, und dann sind die Potenzen von $m$ in einer ganz bestimmten Weise zu erklären. Ist z. B. $m$ negativ, so ist in der Formel (12.) an Stelle von $m^{\frac{2 h+1}{2}-q}$ zu setzen:

$$
m^{\frac{2 h+1}{2}}(-m)^{-q}=(-1)^{\frac{2 h+1}{2}}(-m)^{\frac{2 h+1}{2}-q},
$$

wo das Zeichen $(-1)^{\frac{2 h+1}{2}}$ in demselben Sinne zu nehmen ist, wie in der nunmehr auch zweideutigen Function $J^{\frac{2 h+1}{2}}(m r)$. Für negative Werthe von $m$ werden also die Formeln (13.), (14.):

$$
\begin{aligned}
& \int_{0}^{\infty} J^{\left(\frac{2 h+1}{2}\right)}(m r) d r=(-1)^{\frac{2 h+1}{2}} \sqrt{\frac{1}{2} \pi} \cdot \frac{1}{-m}, \\
& \int_{0}^{\infty} J^{\left(\frac{2 h+1}{2}\right)}(m r) \frac{d r}{r}=(-1)^{\frac{2 h+1}{2}} \sqrt{\frac{1}{2} \pi} \cdot \frac{2}{2 h+1},
\end{aligned}
$$

während für $m=0$ beide Integrale verschwinden. 
Ich hebe endlich noch einen dritten speciellen Fall hervor, den man durch die Annahme $q=h+1$ erhält. Ersetzt man dann in der Formel (12.). die Functionen $J^{\left(\frac{2 h+1}{2}\right)}$ durch die Function $S^{(h)}$, so ergiebt sich:

$$
\begin{cases}\int_{1}^{\infty} \frac{S^{(h)}(m r)}{r} d r=\frac{1}{2} \pi \frac{1.3 .5 \ldots h-1}{2.4 .6 \ldots h} & h \text { gerade } \\ \int_{i}^{\infty} \frac{S^{(h)}(m r)}{r} d r=\frac{2.4 .6 \ldots h-1}{1.3 .5 \ldots h} & h \text { ungerade. }\end{cases}
$$

Diese Integrale gehen für die Fälle $h=0$ und $h=1$ in bekannte Integrale über; sie sind, wie man sieht, für $m=0$ unstetig, und zwar so, dass zu beiden Seiten der Unstetigkeit bei geradem $h$ die entgegengesetzten, bei ungeradem die nämlichen Werthe stattinden.

Heidelberg, im Januar 1868. 